\title{
Continuous Flash Suppression Inhibits Semantic Processing of Chinese Characters with Unawareness: An Event-Related Potential Study
}

\author{
Yiran Lang, Ming Gao, Rongyu Tang \\ Beijing Advanced Innovation Center for Intelligent Robot \& System, Beijing Institute of Technology, Beijing, China \\ Email: yiran.lang@bit.edu.cn
}

How to cite this paper: Lang, Y.R., Gao, M. and Tang, R.Y. (2018) Continuous Flash Suppression Inhibits Semantic Processing of Chinese Characters with Unawareness: An Event-Related Potential Study. Journal of Behavioral and Brain Science, 8, 574-586. https://doi.org/10.4236/jbbs.2018.810035

Received: September 13, 2018

Accepted: October 21, 2018

Published: October 24, 2018

Copyright (c) 2018 by authors and Scientific Research Publishing Inc. This work is licensed under the Creative Commons Attribution International License (CC BY 4.0). http://creativecommons.org/licenses/by/4.0/

\begin{abstract}
Semantic processing is a high level cognition function of the human brain. It has been argued intensively whether this process could be accomplished in the absence of awareness. Continuous flash suppression (CFS) is a powerful approach to create stable unconscious state. In this study, subjects were required to observe a series of paired Chinese words and judge the correlation between them in CFS paradigm. A negative component of event related potential, N400, and a Brain symmetry index were used to measure whether the meaning of the paired stimulus was registered in subliminal semantic processing. Analysis didn't detect a significant difference in N400 response and EEG signal symmetry between correlated and uncorrelated pairs under unconscious condition. These results support the view that semantic information of Chinese character processing is absent without consciousness created by CFS.
\end{abstract}

\section{Keywords}

Continuous Flash Suppression, Semantic Processing, Awareness, N400

\section{Introduction}

Semantic processing of words is a high-level processing of human visual function. Traditionally, people hold that it requires awareness to recognize and analyze the relation of words. For instance, many experiments demonstrate that high-level visual information, i.e. faces and words could not be processed without awareness under visual suppression [1] [2].

Nevertheless, there is controversy concerning whether semantic visual information could be processed unconsciously. People argue that subliminal visual 
stimuli might influence decision making of brand choice [3]. Similarly, many other researchers hold that semantic processing exists under an unconscious state. Costello P. found that related priming words assisted target words breaking visual suppression faster than unrelated pairs [4]. Sklar A. Y. [5] has shown that subliminal priming not only affected understanding of multi-word expression, but also facilitated abstract mathematical computation.

To study subliminal semantic processing, it is required to access an unconscious visual state during the experiment. Interocular suppression provides an effective mean to create an unconscious state for visual stimulation [6] [7]. It is also utilized to study to which extend and to which brain area the visual stimuli could be delivered and processed without awareness [8] [9]. Continuous flash suppression (CFS) is a variant of interocular suppression which produces stable and deep suppression caused by accumulated effects of multiple flashes [10] [11].

Kutas and his colleague found a negative ERP (event-related potential) component, N400, which would appear around $\mathrm{Fz} \mathrm{Cz} \mathrm{Pz}$ position $400 \mathrm{~ms}$ after the onset of detection of unmatched word pairs [12]. Visual sensation of ambiguous, unrelated, non-word, or new word pairs elicits N400 component [13] [14]. It is utilized to objectively reflect brain reaction to such a word pair in linguistics and psychology research. An experiment supports that subliminal presentation of English word pair was not sufficient to induce semantic processing by measuring ERP N400 [15]. Comparing with Latin word, Chinese word expresses not only pronunciation attribute, but also its meaning from its appearance. A behavioral result shows that emotion related Chinese character breaks suppression slower than other groups, which implies that there might be subliminal processing of Chinese character [16].

Nonetheless, EEG evidence is still lacking whether there is semantic processing for Chinese word pair under unconscious state. In the present study, we measured N400s and a brain symmetry index to clarify if Chinese word visual stimulus is processed in the absence of awareness.

\section{Materials and Methods}

\subsection{Ethics Statement}

The experimental procedure was approved by the Beijing Institute of Technology, Beijing, and by the governmental regulations of China. All the participants gave written informed consent before starting the experiment which was approved by the Academy of Beijing Institute of Technology's academic committee.

\subsection{Subjects}

Thirteen healthy volunteers (5 females, age range 22 - 30 years) with normal or corrected-to-normal vision participated in the experiment and were paid for participation. The whole procedure takes 3.5 hours to finish for each participant. 
All the tests were completed in two months. Person who has amblyopia, hypochromatopsia, or problem with visual stereopsis will not be recruited. All participants graduated from university. They were well educated with Chinese characters and were right hand dominant. Their eye dominance was measured according to Yang et al. [16] before the experiment.

\subsection{Apparatus}

Stimuli of Chinese character and Mondrian image were generated by C++ language and demonstrated with STIM2 (Neuroscan, USA) on a HP compad dc7100 desktop computer with a 21-in. LG L1953T monitor. The refresh frequency was $75 \mathrm{~Hz}$ under $1080 \times 1024$ resolutions, and luminance of background was $0.6 \mathrm{~cd} / \mathrm{m}^{2}$. Screen of monitor was viewed at a distance of $60 \mathrm{~cm}$ in a dark room. Binocular vision was formulated through a set of homemade stereotypic glasses which were composed of four pieces of glasses (Figure 1). The two in the middle were $+/-45^{\circ}$ to the sagittal plane and the other two were adjustable to reflect mask and target (to-be-suppressed) image to corresponding eyes. Response to stimuli was received by a HP USB keyboard. Electroencephalogram [17] signals were obtained from $\mathrm{Ag} / \mathrm{AgCl}$ electrodes embedded in elastic woven material EEG cap (NeuroscanQuik-Cap, USA). The location of electrodes on cap was consistent with international 10-20 system. Signals in position $\mathrm{Fz}, \mathrm{Cz}, \mathrm{Pz}$, F3, F4, C3, C4, P3, P4, PO3, PO4, O1, O2, F7, F8, FT7, FT8, T7, T8, P7, and P8 were recorded. These electrodes were referenced to the right mastoid and grounded to forehead. Sampling frequency was $1000 \mathrm{~Hz}$ and the gain of the signal was 20,000 and bandpass was $0.01-100 \mathrm{~Hz}$.

\subsection{Stimuli}

Visual stimuli were composed of a primer and a target double-character Chinese word $\left(1.68^{\circ} \times 1.68^{\circ}\right)$ that randomly appeared above or below a central fixation

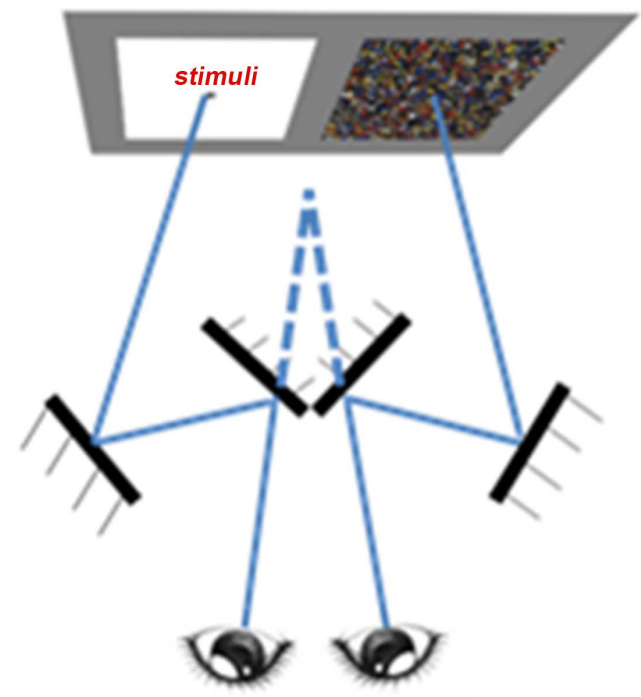

Figure 1. Stereotaxic glasses and vision of stimuli. 
cross (black, $0.34^{\circ} \times 0.34^{\circ}$ ). The contrast of target stimuli was $25 \%$ on a white background and the primer was $100 \%$. Font of the words was the song typeface. A word pool with 240 frequently used words was chosen from "Mordern Chinese frequency dictionary" (1986). The word frequency ranged from 0.0025 to 0.0057. Among them, 60 pairs were selected as related (REL) group and the other 60 pairs were defined as unrelated (UNR). For instance, 秋天 (autumn) and 丰收 (harvest) were a correlated pair. The correlation score of primer and target were evaluated by six graduate students who didn't participate in this experiment according to a seven-level measuring scale. Only word pairs biased strongly to related or unrelated would be used as stimuli.

To create interocular suppression, a series of Mondrian patterned images (composed of $0.1^{\circ}-0.35^{\circ}$ colored squares with random RGB color ranging from 0 to 255 respectively. The size and position of squares were stochastic. Overlay was acceptable.) were generated and presented to the dominant eye. All the colored squares were contained in a $5.06^{\circ} \times 6.4^{\circ}$ frame. Four Mondrian images were presented in each trial in a random sequence with $10 \mathrm{~Hz}$ refresh rate $(100$ ms for each image). Target and Mondrian mask images were surrounded by $14.16^{\circ} \times 9.10^{\circ}$ grey square background $(128,128$, and 128 in RGB, Figure 2).

\subsection{Procedure}

Participants were introduced to sit on a chair comfortably with head laid on a headrest to ensure the consistency of distance between eyes and screen of the monitor to be $60 \mathrm{~cm}$. There were 10 extra trials for them to practice before the main experiment. Words used in extra trials would not appear in the experiment. In the beginning of the experiment, central fixation points in left and right images were shown for $2000 \mathrm{~ms}$ to make participants focus and fuse both images as perfect as possible. In each trial, primer words were presented first to both eyes for $1000 \mathrm{~ms}$. Along with the third Mondrian image, the target stimulus were shown to the non-dominant eye with contrast 25\% and the masking Mondrian pattern shown to the other eye switched randomly every $100 \mathrm{~ms}(10 \mathrm{~Hz})$ after trial onset. $500 \mathrm{~ms}$ post end of Mondrian sequence, participants were asked to report their perception of correlation of words pairs by clicking number 4 or 6 on the keyboard regardless of targets being seen consciously or not. Response time (RT) was not restricted, but RT more than 4 seconds would be ruled out of analysis. Subsequent trial would be initialized once response was made.

\subsection{Brain Symmetry Index}

N400 component distribution is asymmetrical among all electrodes depending on stimuli type and brain area involved in the process. Chinese character is a compound of pronouncing, font face, and meaning, which might require various brain areas to function simultaneously. To this end, brain symmetry index (BSI) was used to quantitatively measure the symmetry of EEG signal distribution [18] [19] [20] by comparing left and right electrode pairs. Firstly, average spectral 


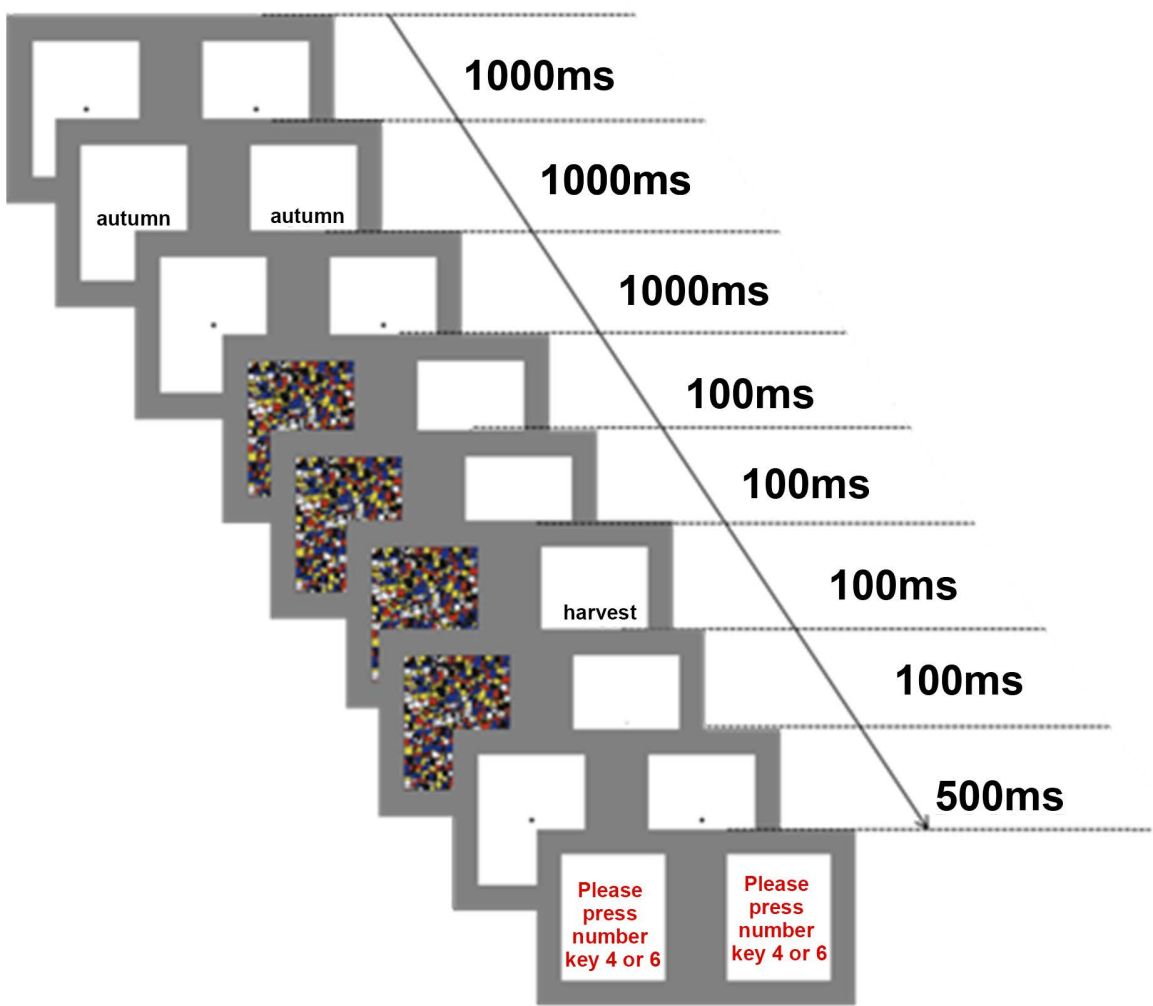

Figure 2. Illustration of visual stimuli sequence of a trial. Primer word was presented to both eyes in identical position in each frame after $1000 \mathrm{~ms}$ of fixation. Four Mondrian images were presented to one eye in turn after another $1000 \mathrm{~ms}$. Target word (丰收, means harvest) was shown to the other eye for $100 \mathrm{~ms}$ with the third Mondrian image. A $500 \mathrm{~ms}$ period was followed by behavior judgment session which required subjects to choose REL or UNR by pressing number key 4 or 6 to indicate.

density of EEG signals ranged from 0 to $35 \mathrm{~Hz}$ was calculated. We wrote for the absolute power of signal obtained from a paired channel $i=1, \cdots, N$, from left $\left(L_{i j}(t)\right)$ and right $\left(R_{i j}(t)\right)$ hemisphere at Fourier coefficient $j=1, \cdots, M$. Thus, now BSI could be defined as:

$$
B S I(t)=\left\|\frac{1}{N} \frac{1}{M} \sum_{i=1}^{M} \sum_{j=1}^{M} \frac{R_{i j}(t)-L_{i j}(t)}{R_{i j}(t)+L_{i j}(t)}\right\|
$$

with $N$ the number of electrode pairs and $\mathrm{M}$ the value of Fourier coefficients. Distribution of all the electrodes is normalized and integrated into the BSI. The maximum of BSI would be 1 , implied completely asymmetry, whereas the minimum of BSI was 0 , referred to perfect symmetry for all channels.

\subsection{Analysis}

The signals were further low-pass filtered offline using EEGLAB (version 13.4b, basic FIR filter with 0 - $35 \mathrm{~Hz}$ for both end of frequency end). We manually reviewed and discarded the trials with eye movement, eye closure, or nearby muscle noise. The point of origin of the ERP signal was the onset of the target. The signal prior to target served as baseline $(-200-0 \mathrm{~ms})$. The sampling rate was 
$1000 \mathrm{~Hz}$. Waveforms were band pass filtered $(0-35 \mathrm{~Hz})$. It was considered significantly different when $\mathrm{p}<0.05$.

\section{Results}

We created an unconscious state for subjects while watching related or unrelated Chinese word pairs. Participants had an obligation to response related and unrelated words by pressing two buttons. Reaction Time (RT) longer than $4 \mathrm{~s}$ would be excluded from data analysis. Mean RT for REL trials was 637.41 \pm $306.96 \mathrm{~ms}$ (mean $\pm \mathrm{SD}$ ), and for UNR trials was $690.23 \pm 303.82 \mathrm{~ms}$. There was no significant differences between them (paired t-test, $\mathrm{t}=-1.816, \mathrm{p}=0.094$ ). It indicated that relationship of primer and target did not influence the RT of subjects.

Accuracy of behavioral response of all the subjects ranged between $43.64 \%$ and $95.45 \%(67.83 \% \pm 16.32 \%)$. In detail, response accuracy for related word pairs was $58.04 \% \pm 21.33 \%$, and for unrelated pairs was $77.62 \% \pm 21.6 \%$. Among them, accuracy for related pairs of two subjects was $>90 \%$. For unrelated pairs, however, accuracy of eight subjects was $>90 \%$. It implied that they might be aware of the meaning of target words even under CFS suppression. Paired t-test found that accuracy for unrelated word pairs was significantly higher than related pairs $(\mathrm{t}=-2.433, \mathrm{p}=0.032)$, indicating that unconsciousness created by CFS might be more effective for related word pairs than unrelated pairs. Two tailed one sample t-test showed that P-value of behavioral response accuracy of other trials $(52.58 \% \pm 3.15 \%$, mean $\pm \mathrm{SE})$ was 0.431 comparing with $50 \%$. Thus, accuracy of these trials was not significantly different from the chance level which showed that target words were suppressed effectively. The results of participants who were aware of the meaning of target words would be defined as the control group (Control), while the results under unconscious state served as the experimental group (Suppresion).

Figure 3(a) and Figure 3(d) showed the averaged ERPs of three vital electrode $\mathrm{Fz}, \mathrm{Cz}$, and $\mathrm{Pz}$ across 5 subjects for experimental group and 8 subjects for control group. A trough appeared after the onset of target words and followed by a rising period approximate $200 \mathrm{~ms}$. The waveforms showed different trend for experimental and control group. While participants in control group awared of the target words consciously, the ERP waveforms ignited by unrelated word pairs were obvious higher than those by related word pairs. On the other hand, waveforms of suppressed state behaved similarly for REL and UNR. Topograh (Figure 3(b) and Figure 3(e)) demonstrated the divergent trend of N400 of all the 21 electrode positions under suppressed and awared state. Under suppression, voltage of occipital and temporal lobe kept constant, along with parietal lobe enhancing slight. But voltage of frontal lobe showed obvious decreasing. On the contrary, voltage of almost every electrode of control group decreased, especially in parietal and temporal lobe.

The amplitude of N400 (obtained from the voltage difference for REL and UNR ranging from $350 \mathrm{~ms}$ to $500 \mathrm{~ms}$ post onset of target words) of three vital 


\section{Suppression}

(a)
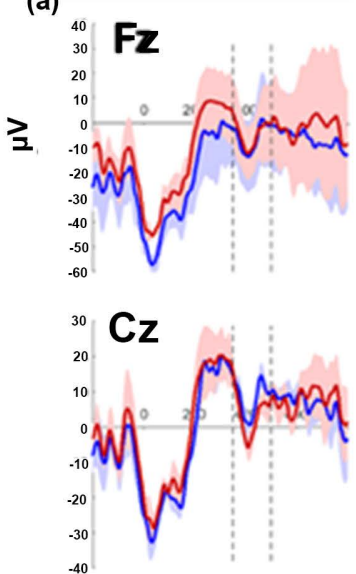

$\mathrm{Pz}$

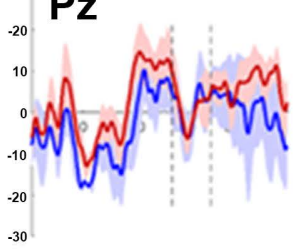

(b)

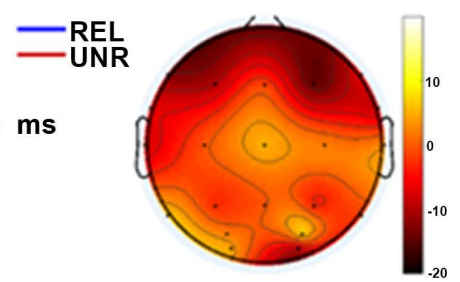

(c)

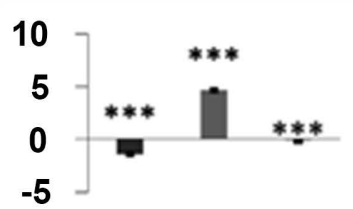

Fz $\quad \mathrm{Cz} \quad \mathrm{Pz}$ (d)
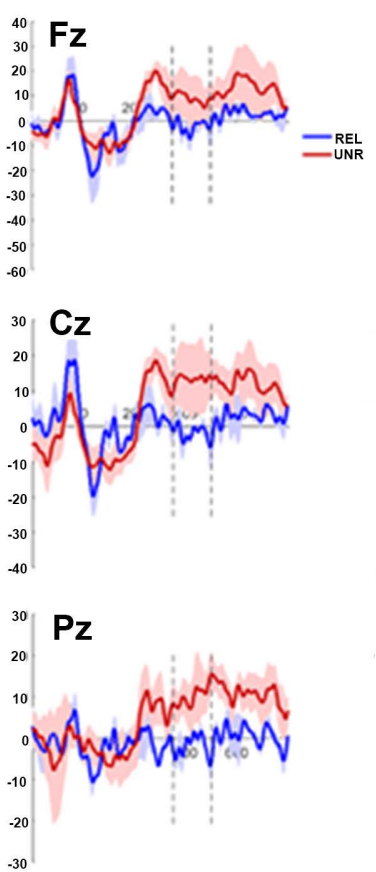

Control

(e)

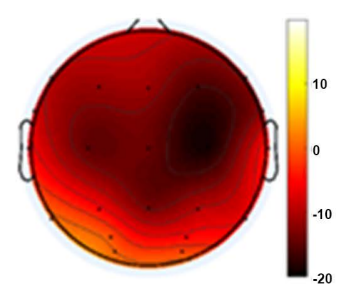

(f)

Fz $\quad \mathrm{Cz} \quad \mathrm{Pz}$

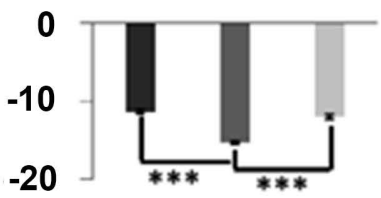

Figure 3. Results of ERP. (a) Waveforms of ERP under suppressed state (suppression) and (d) aware state (control) of target words. Blue line depicts waveforms of ERP stimulated by related word pairs (REL) and those elicited by unrelated pairs are in red (UNR). Shaded area of corresponding color around ERP waveforms is \pm 1 S.E. of signals. Upper graph is the waveforms of electrode Fz. Middle is $\mathrm{Cz}$ and bottom is Pz. Dotted vertical lines indicate the temporal window of N400 ranging between 350 - $500 \mathrm{~ms}$ after target words appear. (b) and (e) are topographs of the difference (REL - UNR) of all electrode averaged across subjects during the $350 \mathrm{~ms}-500 \mathrm{~ms}$ temporal window. Darker end of color bar refers to $-20 \mu \mathrm{V}$ and yellow end refers to $10 \mu \mathrm{V}$. (c) and (f) are Histogram and S.E. of difference of waveforms of three vital electrodes for related and unrelated word in the temporal window under suppression and control condition. Asterisks indicates significant difference among electrodes $\left({ }^{* *} \mathrm{p}<0.001\right)$.

EEG electrodes were compared with one way ANOVA test. Post hoc multiple comparisons (Bonferroni) demonstrated that the difference of waveforms of the three electrodes of suppression state were significantly different $(\mathrm{p}<0.001)$ from each other. The value of $\mathrm{Pz}(-0.1888 \pm 0.1639 \mu \mathrm{V})$ was higher than $\mathrm{Fz}(-1.422 \pm$ $0.3007 \mu \mathrm{V})$ but lower than $\mathrm{Cz}(4.6643 \pm 0.16 \mu \mathrm{V})$. For the control group, on the contrary, voltage of $\mathrm{Cz}$ was much lower $(-15.306 \pm 0.182 \mu \mathrm{V})$ than $\mathrm{Fz}(-11.3944$ $\pm 0.2455 \mu \mathrm{V})$ and $\mathrm{Pz}(-11.9804 \pm 0.2829 \mu \mathrm{V})(\mathrm{p}<0.001$, Figure 3$)$. There was no significant difference between $\mathrm{Fz}$ and $\mathrm{Pz}(\mathrm{p}=0.256)$. These findings suggested that incomplete suppressing of target words result in different response of waveform of each vital electrodes to related and unrelated word pairs. Unrelated word pairs elicited higher voltage in N400 component than related words. Waveforms of the three electrodes were significantly different in suppression and control group $(\mathrm{p}<0.001)$.

Since the reaction of ERP waveform to related and unrelated word pairs differed between suppression and control condition. We asked whether each frequency component of EEG signal behaved in concert with the trend of ERP. Af- 
ter spreading up frequency spectrum, power of three frequencies, $5 \mathrm{~Hz}, 8 \mathrm{~Hz}$, and $15 \mathrm{~Hz}$ were analyzed representing theta, alpha, and beta band (Figure 4). Waveforms of the three frequencies elicited by related and unrelated word pairs were depicted and differences between 350 - $500 \mathrm{~ms}$ after target word onset were compared. For $5 \mathrm{~Hz}$, power for REL pairs was stronger than it elicited by UNR pairs. Mean difference of control group was $0.194 \pm 0.014 \mu \mathrm{V}^{2}$ (mean \pm S.E.), meanwhile, suppression group was $0.398 \pm 0.005 \mu \mathrm{V}^{2}$. In contrast, under suppression state, power ignited by REL in $8 \mathrm{~Hz}$ and $15 \mathrm{~Hz}$ were much lower than it by UNR which resulted in negative difference $\left(-0.172 \pm 0.028 \mu \mathrm{V}^{2}\right.$ for $8 \mathrm{~Hz}$, $-0.077 \pm 0.006 \mu \mathrm{V}^{2}$ for $15 \mathrm{~Hz}$ ). It was in sharp contrast with control group in which the differences were still positive $\left(0.135 \pm 0.010 \mu \mathrm{V}^{2}\right.$ for $8 \mathrm{~Hz}, 0.350 \pm$

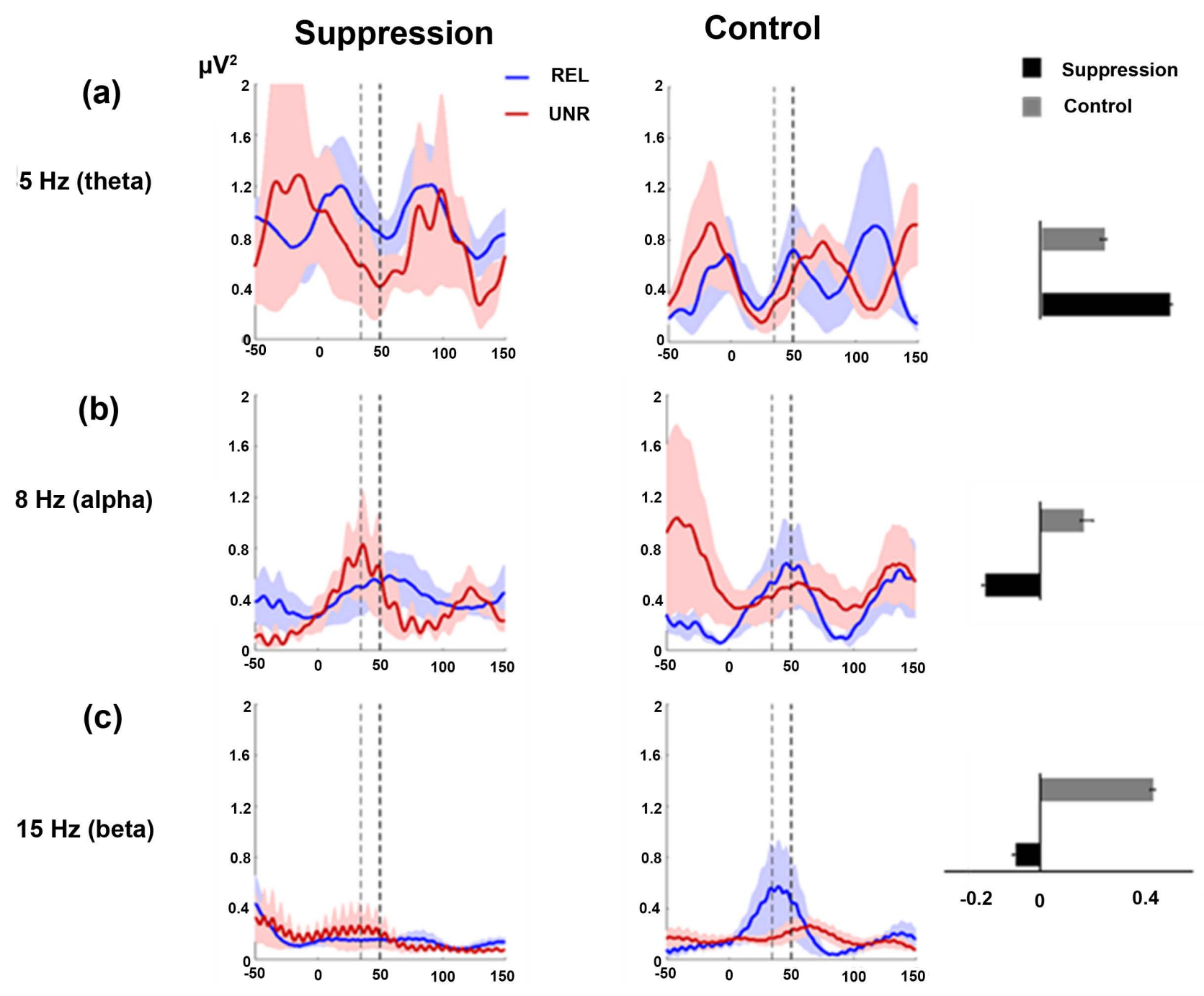

Figure 4. EEG power of $\mathrm{Cz}$ of three main frequencies. Blue and red lines depict power mined in ERPs elicited by related word pairs and unrelated pairs respectively of (a) $5 \mathrm{~Hz}$, (b) $8 \mathrm{~Hz}$, and (c) $15 \mathrm{~Hz}$. Shaded area of corresponding color around EEG power is \pm 1 S.E. of averaged power. Dotted vertical line indicates the temporal window of N400 ranging between $350-500 \mathrm{~ms}$ after target words appear. Left and middle graphs of each frequency demonstrate EEG power under suppression and control conditions in $\mu \mathrm{V}^{2}$. Right histogram along with EEG power is the difference of power of each frequency in the temporal window (350-500 ms post target onset). Black bar is the suppression group and grey bar is the control group. 
$0.010 \mu \mathrm{V}^{2}$ for $15 \mathrm{~Hz}$ ). T-test demonstrated that the differences of all the suppression groups was significant higher than control groups $(\mathrm{p}<0.001)$.

Unbalanced variant trend of EEG activity in each hemisphere was observed in Figure 3. In control group, N400 difference between REL and UNR was larger in right frontal and parietal lobes. To investigate the asymmetry of distribution of EEG activity during N400 period (averaged over 100 - $500 \mathrm{~ms}$ post target words onset), we measured BSI of all the electrodes during $1000 \mathrm{~ms}$ before and $2000 \mathrm{~ms}$ after the onset of the target word (Figure 5). In suppression condition, baseline of BSI was $0.203 \pm 0.011$ for REL and $0.207 \pm 0.007$ for UNR, which was equal to the value of $100 \sim 500$ ms periods ( $p=0.282$ and 0.776 ). Similarly, BSI in control condition was not significantly changed before and after the target word onset ( $\mathrm{p}$ $=0.517$ and 0.231 for REL and UNR). Nonetheless, BSI during the $100 \sim 500 \mathrm{~ms}$ periods showed a significant difference between REL and UNR of control condition, but not suppression condition $(\mathrm{p}=0.015)$.

\section{Discussion}

The present study adopted CFS paradigm, a variant of binocular rivalry, to examine whether the meaning of single Chinese word can be processed unconsciously. By using CFS paradigm and measuring accuracy of response, partial awareness state is clearly identified and defined as control group. N400 in

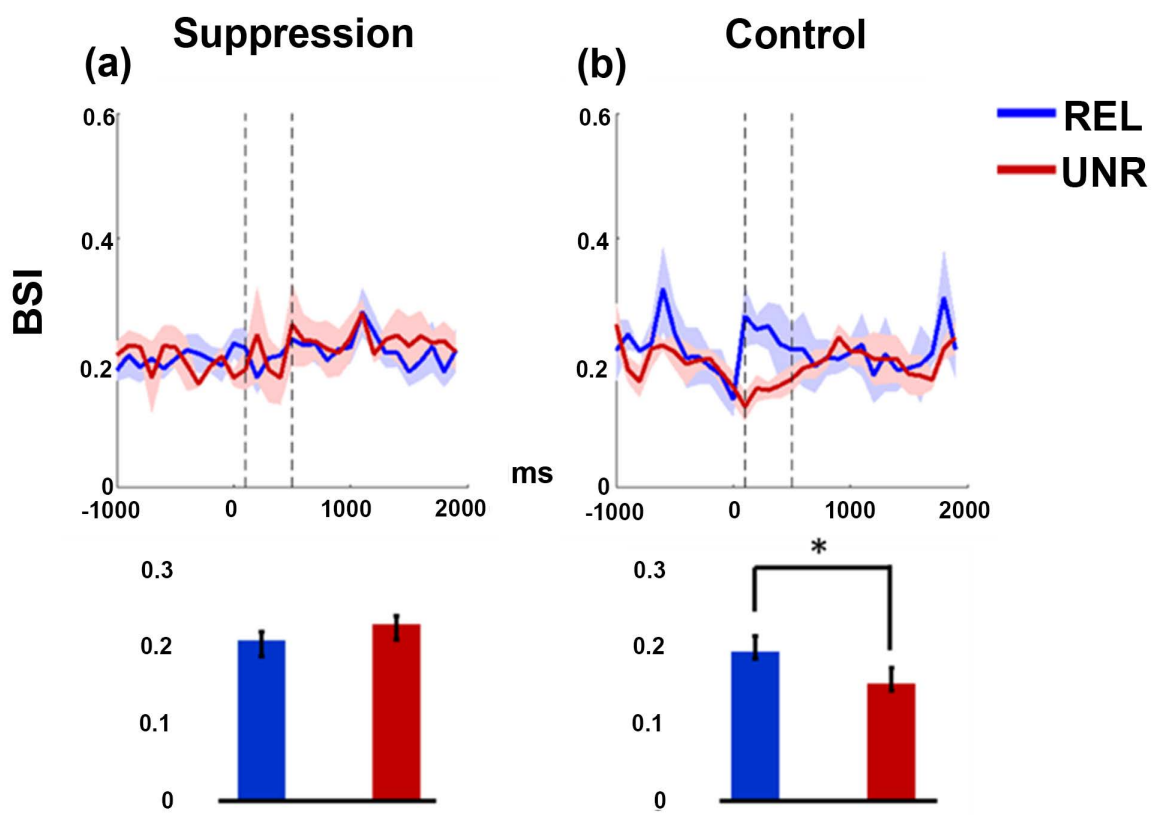

Figure 5. BSI of ERP. (a) Upper graph, BSI of signals under suppression condition. Blue and red lines refer to BSI of all electrodes along with experimental timeline elicited by related word pairs (REL) and unrelated pairs (UNR) respectively. Shaded area of corresponding color around BSI is \pm 1 S.E of mean value. Dotted vertical line indicates the temporal window of N400 ranging between 100 - $500 \mathrm{~ms}$ after target words appear. Lower graph, histogram of averaged BSI among 100 - 500 ms post target words onset. (b) BSI under the control condition. Asterisks indicates a significant difference among electrodes $\left({ }^{*} \mathrm{p}<0.05\right)$. 
combination with BSI results converges in which neural activity of unconscious condition differs from aware condition. Thus, it can be deduced that no subliminal semantic processing of single Chinese word exists under unconscious condition during $\mathrm{N} 400$ period.

Studies regarding N400 demonstrate that potentials of EEG electrodes in each hemisphere are not symmetrically distributed [13] [21] [22]. Consistent with Kutas, et al. [22] results, our experiment shows that unrelated words elicit stronger potentials on the right hemisphere, but not related words, even if left hemisphere is considered significant in language comprehension. In suppression condition, this phenomenon is attenuated by continuous flash suppression. Right hemisphere is also important in noticing anomalous words in priming context [23]. Another experiment finds that both hemispheres exhibit similar responses to different ranges of message constraint sentences, more or less predictable in N400 component [24]. An fMRI study shows that invisible words or sentences could be discriminated in left posterior superior temporal sulcus and left middle frontal gyrus [25]. These results may suggest that both hemispheres are critical in language expectation and comprehension but right hemisphere might involve more in processing of unexpected or unrelated word. Thus, brain activity distribution symmetry is a possible index depicting processing of semantic analysis taking place in visual pathway.

We presume that the different distributions of neural representation are resulted from the difference between Latin and Mandarin language in the beginning. Diverse structure of Chinese character elicits split reaction in two hemispheres which indicate that semantic and phonetic parts of a character are processed separately [26]. However, fMRI and MEG studies find that English or German sentence was processed in left temporal sulcus, middle temporal area, and left middle frontal gyrus [25] [27]. For the case of Mandarin, visual naming and word making tasks activate left fusiform gyrus, left middle frontal gyrus, and left superior temporal gyrus [28]. The brain areas recruited for Latin and Mandarin language are similar. Another MRI study supports that, during translation between English and Chinese words, neural representation among different people is conservative [29]. Thus, the diversity of neural activity distribution and asymmetry is derived from different tasks and stage of information processing.

In the current study, power of theta frequency of $\mathrm{Cz}$ demonstrates stronger reaction to related word pairs than unrelated counterparts. Recent research has demonstrated that different ranges of power bands response differently to semantic stimuli. Grammatical error sentence induces beta power decrease in adult subjects [30]. Theta frequency may reflect interaction of semantic processing and working memory, while gamma band indicates activation of neural network for semantic analysis [31]. Alpha power decreases when emotional words are perceived [32] and desynchronized when well-known knowledge is violated, but not theta or gamma band [33]. These results indicate that not only ERPs, but also time-frequency components correlate with semantic in- 
formation processing.

The current study requires participants to recognize relationship between primer and target word. The single Chinese word fails to break the interocular suppression. However, we cannot rule out the possibility that early stage unconscious processing still exists. It is worth studying whether more salient stimulus, like emotional word or complete sentence, break the suppression and enter awareness or not using electrophysiology techniques.

\section{Acknowledgements}

This work was supported by the State Key Program of National Natural Science Foundation of China (Grant Nos. 91648207), the National Natural Science Foundation of China (Grant Nos. 61673068), and Technology R \& D Program of Beijing (Grant Nos. Z181100003118007).

\section{Conflicts of Interest}

The authors declare no conflicts of interest regarding the publication of this paper.

\section{References}

[1] Moradi, F., Koch, C. and Shimojo, S. (2005) Face Adaptation Depends on Seeing the Face. Neuron, 45, 169-175. https://doi.org/10.1016/j.neuron.2004.12.018

[2] Heyman, T. and Moors, P. (2014) Frequent Words Do Not Break Continuous Flash Suppression Differently from Infrequent or Nonexistent Words: Implications for Semantic Processing of Words in the Absence of Awareness. PLOS ONE, 9, e104719. https://doi.org/10.1371/journal.pone.0104719

[3] Karremans, J.C., Stroebe, W. and Claus, J. (2006) Beyond Vicary's Fantasies: The Impact of Subliminal Priming and Brand Choice. Journal of Experimental Social Psychology, 42, 792-798. https://doi.org/10.1016/j.jesp.2005.12.002

[4] Costello, P., Jiang, Y., Baartman, B., McGlennen, K. and He, S. (2009) Semantic and Subword Priming during Binocular Suppression. Consciousness and Cognition, 18, 375-382. https://doi.org/10.1016/j.concog.2009.02.003

[5] Sklar, A.Y., Levy, N., Goldstein, A., Mandel, R., Maril, A. and Hassin, R.R. (2012) Reading and Doing Arithmetic Nonconsciously. Proceedings of the National Academy of Sciences of United States of America, 109, 19614-19619. https://doi.org/10.1073/pnas.1211645109

[6] Blake, C.-Y. and Blake, R. (2005) Psychophysical Magic: Rendering the Visible "Invisible". Trends in Cognitive Sciences, 9, 8. https://doi.org/10.1016/j.tics.2005.06.012

[7] Lin, Z.C. and He, S. (2009) Seeing the Invisible: The Scope and Limits of Unconscious Processing in Binocular Rivalry. Progress in Neurobiology, 87, 17. https://doi.org/10.1016/j.pneurobio.2008.09.002

[8] Lee, S.H., Blake, R. and Heeger, D.J. (2007) Hierarchy of Cortical Responses Underlying Binocular Rivalry. Nature Neuroscience, 10, 1048-1054. https://doi.org/10.1038/nn1939

[9] Sterzer, P. and Rees, G. (2008) A Neural Basis for Percept Stabilization in Binocular Rivalry. Journal of Cognitive Neuroscience, 20, 389-399. https://doi.org/10.1162/jocn.2008.20039 
[10] Tsuchiya, N. and Koch, C. (2005) Continuous Flash Suppression Reduces Negative Afterimages. Nature Neuroscience, 8, 1096-1101. https://doi.org/10.1038/nn1500

[11] Tsuchiya, N., Koch, C., Gilroy, L.A. and Blake, R. (2006) Depth of Interocular Suppression Associated with Continuous Flash Suppression, Flash Suppression, and Binocular Rivalry. Journal of Vision, 6, 1068-1078. https://doi.org/10.1167/6.10.6

[12] Kutas, M. and Hillyard, S.A. (1980) Reading Senseless Sentences: Brain Potentials Reflect Semantic Incongruity. Science, 207, 203-205. https://doi.org/10.1126/science.7350657

[13] Kutas, M. and Federmeier, K.D. (2011) Thirty Years and Counting: Finding Meaning in the N400 Component of the Event-Related Brain Potential (ERP). Annual Review of Psychology, 62, 621-647. https://doi.org/10.1146/annurev.psych.093008.131123

[14] Lau, E.F., Phillips, C. and Poeppel, D. (2008) A Cortical Network for Semantics: (De)Constructing the N400. Nature Reviews Neuroscience, 9, 920-933. https://doi.org/10.1038/nrn2532

[15] Kang, M.-S., Blake, R. and Woodman, G.F. (2011) Semantic Analysis Does Not Occur in the Absence of Awareness Induced by Interocular Suppression. The Journal of Neuroscience, 31, 11. https://doi.org/10.1523/JNEUROSCI.1691-11.2011

[16] Yang, Y.H. and Yeh, S.L. (2011) Accessing the Meaning of Invisible Words. Consciousness and Cognition, 20, 223-233. https://doi.org/10.1016/j.concog.2010.07.005

[17] Alex Polonsky, R.B., Braun, J. and Heeger, D.J. (2000) Neuronal Activity in Human Primary Visual Cortex Correlates with Perception during Binocular Rivalry. Nature Neuroscience, 3, 7. https://doi.org/10.1038/80676

[18] van Putten, M.J. and Tavy, D.L. (2004) Continuous Quantitative EEG Monitoring in Hemispheric Stroke Patients Using the Brain Symmetry Index. Stroke, 35, 2489-2492. https://doi.org/10.1161/01.STR.0000144649.49861.1d

[19] van Putten, M.J. (2007) The Revised Brain Symmetry Index. Clinical Neurophysiology, 118, 2362-2367. https://doi.org/10.1016/j.clinph.2007.07.019

[20] Xin, X., Chang, J., Gao, Y. and Shi, Y. (2016) Correlation between the Revised Brain Symmetry Index, an EEG Feature Index, and Short-Term Prognosis in Acute Ischemic Stroke. Journal of Clinical Neurophysiology, 34, 162-167.

[21] Kutas, M. (2000) Electrophysiology Reveals Semantic Memory Use in Language Comprehension. Trends in Cognitive Sciences, 4, 8. https://doi.org/10.1016/S1364-6613(00)01560-6

[22] Kutas, M., Van Petten, C. and Besson, M. (1988) Event-Related Potential Asymmetries during the Reading of Sentences. Electroencephalography and Clinical Neurophysiology, 69, 218-233. https://doi.org/10.1016/0013-4694(88)90131-9

[23] Metusalem, R., Kutas, M., Urbach, T.P. and Elman, J.L. (2016) Hemispheric Asymmetry in Event Knowledge Activation during Incremental Language Comprehension: A Visual Half-Field ERP Study. Neuropsychologia, 84, 252-271. https://doi.org/10.1016/j.neuropsychologia.2016.02.004

[24] DeLong, K.A. and Kutas, M. (2016) Hemispheric Differences and Similarities in Comprehending More and Less Predictable Sentences. Neuropsychologia, 91, 380-393. https://doi.org/10.1016/j.neuropsychologia.2016.09.004

[25] Axelrod, V., Bar, M., Rees, G. and Yovel, G. (2015) Neural Correlates of Subliminal Language Processing. Cerebral Cortex, 25, 2160-2169. https://doi.org/10.1093/cercor/bhu022

[26] Hsiao, J.H., Shillcock, R. and Lee, C.Y. (2007) Neural Correlates of Foveal Splitting 
in Reading: Evidence from an ERP Study of Chinese Character Recognition. Neuropsychologia, 45, 1280-1292.

https://doi.org/10.1016/j.neuropsychologia.2006.10.001

[27] Maess, B., Mamashli, F., Obleser, J., Helle, L. and Friederici, A.D. (2016) Prediction Signatures in the Brain: Semantic Pre-Activation during Language Comprehension. Frontiers in Human Neuroscience, 10, 591. https://doi.org/10.3389/fnhum.2016.00591

[28] Ci, H., van Graan, A., Gonzalvez, G., Thompson, P., Hill, A. and Duncan, J.S. (2016) Mandarin Functional MRI Language Paradigms. Brain and Behavior, 6, e00525. https://doi.org/10.1002/brb3.525

[29] Zinszer, B.D., Anderson, A.J., Kang, O., Wheatley, T. and Raizada, R.D. (2016) Semantic Structural Alignment of Neural Representational Spaces Enables Translation between English and Chinese Words. Journal of Cognitive Neuroscience, 28, 1749-1759. https://doi.org/10.1162/jocn_a_01000

[30] Schneider, J.M., Abel, A.D., Ogiela, D.A., Middleton, A.E. and Maguire, M.J. (2016) Developmental Differences in Beta and Theta Power during Sentence Processing. Developmental Cognitive Neuroscience, 19, 19-30.

https://doi.org/10.1016/j.dcn.2016.01.001

[31] Mellem, M.S., Friedman, R.B. and Medvedev, A.V. (2013) Gamma- and Theta-Band Synchronization during Semantic Priming Reflect Local and Long-Range Lexical-Semantic Networks. Brain and Language, 127, 440-451. https://doi.org/10.1016/j.bandl.2013.09.003

[32] Wang, L. and Bastiaansen, M. (2014) Oscillatory Brain Dynamics Associated with the Automatic Processing of Emotion in Words. Brain and Language, 137, 120-129. https://doi.org/10.1016/j.bandl.2014.07.011

[33] Metzner, P., von der Malsburg, T., Vasishth, S. and Rosler, F. (2015) Brain Responses to World Knowledge Violations: A Comparison of Stimulus- and Fixation-Triggered Event-Related Potentials and Neural Oscillations. Journal of Cognitive Neuroscience, 27, 1017-1028. https://doi.org/10.1162/jocn_a_00731 